nabinoids and intracellular signaling. In Endocannabinoids: the brain and body's marijuana and beyond. E.S. Onaivi, T. Sugiura, and V. Di Marzo, editors. CRC Press/Taylor \& Francis Group, London, United Kingdom. 119-131.

17. Di Marzo, V., and De Petrocellis, L. 2006. Non$\mathrm{CB} 1$, non-CB2 receptors for endocannabinoids. In
Endocannabinoids: the brain and body's marijuana and beyond. E.S. Onaivi, T. Sugiura, and V. Di Marzo, editors. CRC Press/Taylor \& Francis Group. London, United Kingdom. 151-174.

18. Ligresti, A., and Di Marzo, V. 2006. Endocannabinoid-based molecules as potential therapeutic drugs. In Endocannabinoids: the brain and body's mari- juana and beyond. E.S. Onaivi, T. Sugiura, and V. Di Marzo, editors. CRC Press/Taylor \& Francis Group. London, United Kingdom. 537-554.

19. Despres, J.P., Golay, A., and Sjostrom, L. 2005. Effects of rimonabant on metabolic risk factors in overweight patients with dyslipidemia. N. Engl. J. Med. 353:2121-2134.

\title{
The glomerular basement membrane: not gone, just forgotten
}

Marilyn G. Farquhar

Department of Cellular and Molecular Medicine, UCSD, La Jolla, California, USA.

\begin{abstract}
The glomerular capillaries function as the filtration barrier that retains albumin and other plasma proteins in the circulation. The unresolved question that has been asked for more than 50 years is, Which structural component of these capillaries constitutes the main molecular sieve that normally retains albumin and allows its passage in diseases associated with proteinuria? There is considerable evidence implicating both the glomerular basement membrane (GBM) and the epithelial filtration slits as the barrier. However, the prevailing point of view at present is that the slit diaphragms bridging the filtration slits are responsible for this important function, and evidence implicating the GBM is largely ignored or forgotten. In this issue of the JCI, Jarad et al. show that in laminin $\beta 2$-deficient (Lamb2-/-) mice, proteinuria can be directly attributed to the altered composition of the GBM (see the related article beginning on page 2272). Changes in the permeability of the GBM and its organization were primary to changes in the epithelium, as they preceded foot process effacement and loss of slit diaphragms.
\end{abstract}

It has been more than 50 years since the early days of EM, when the ultrastructural organization of the glomerular capillary wall was defined. Yet there is still no consensus concerning which component - the slit diaphragms bridging the filtration slits or the glomerular basement membrane $(\mathrm{GBM})$ - represents the primary glomerular filtration barrier. Over the intervening years, the pendulum has swung back and forth according to the interpretation of the evidence available. Since the discovery in 1999 of nephrin, a slit diaphragm protein, and its identification as the defective gene in patients with congenital nephrosis of the Finnish type (CNF) (1), the prevailing view has been that the slit diaphragms located in the filtration slits that attach the adjoining foot processes to one another contain

Nonstandard abbreviations used: GBM, glomerular basement membrane; Lamb2, laminin $\beta 2$.

Conflict of interest: The author has declared that no conflict of interest exists.

Citation for this article: J. Clin. Invest. 116:2090-2093 (2006). doi:10.1172/JCI29488. pores responsible for the molecular sieving that prevents passage of albumin across the capillary wall. The work of Jarad et al. (2) in this issue of the JCI clearly demonstrates that defects in the composition and integrity of the GBM meshwork can lead to proteinuria, thus emphasizing the crucial role of the GBM in filtration and lending support to the concept that the fibrillar meshwork of the GBM functions as the molecular sieve that retains albumin.

\section{The swinging of the pendulum}

The first direct experimental evidence aimed at identification of the glomerular filtration barrier was based on the use of the electron-dense tracer ferritin $(3,4)$ and later, graded dextrans of varying sizes (MW, 32,000-125,000 Da) (5), which pinpointed the GBM as the layer that prevents passage of both of these tracers. Ferritin and dextrans of the size of albumin or greater accumulated against the luminal surface of the GBM. Later on, quite a number of different tracers were used, and most accumulated against the GBM rather than in the epithelial slits. This work was superseded by findings obtained largely by Karnovsky and coworkers using enzymatic tracers of different sizes such as catalase (MW, 220,000 $\mathrm{Da}$ ) and HRP (MW, 40,000 Da), which were detected by a histochemical reaction (reviewed in ref. 6). These studies suffered from serious technical limitations (6), but they were interpreted as demonstrating that the filtration slits form the selective filter. Based on these studies, it was proposed that the GBM is a coarse pre-filter and the epithelial slits function as the crucial molecular sieving layer. This idea was reinforced by the discovery of a periodic structure interpreted as "slit-pores" in the slit membranes bridging the filtration slits (7). Later, Ryan and Karnovsky (8) observed that when renal blood flow was normal, plasma albumin, like ferritin and dextrans, was absent from the GBM and urinary spaces, but large amounts could be detected by immunocytochemistry in both sites if the tissue was immersion fixed or renal blood flow was interrupted by ligation of the renal artery. These studies underlined the importance of the GBM as the protein filter under normal flow conditions and helped to explain some of the authors' earlier results that had been obtained from kidney tissue fixed by immersion. Yet the view that the GBM is a coarse pre-filter and the epithelial slits serve as a fine filter restricting the passage of albumin has lived on and remained predominant in the field.

Based on the work of Brenner and associates on the importance of charge as a determinant of glomerular filtration, interest in the 1970s and 1980s shifted to defining the charge barrier (9). A sialic acid-rich, highly negatively charged cell-surface coat was found on podocytes, and regularly spaced, 


\section{A WT mice}

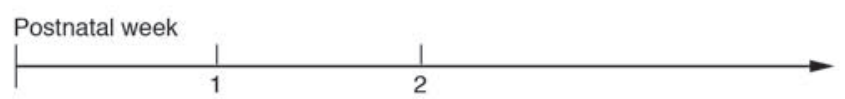

\section{B Lamb2 $2^{--}$mice}

Postnatal week

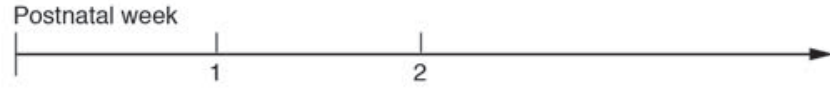

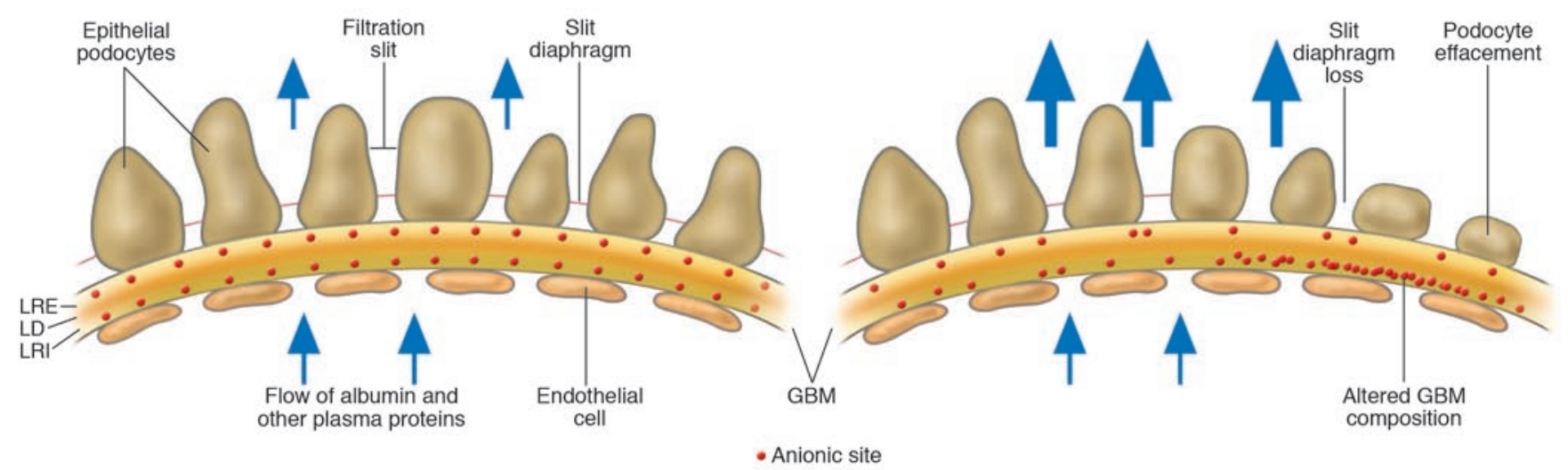

Capillary lumen

Capillary lumen

Figure 1

Diagram of the glomerulus in normal and Lamb2-/- mice. (A) In the normal glomerulus, the anionic sites are concentrated in the lamina rara interna (LRI) and lamina rara externa (LRE) of the GBM and distributed at regularly spaced intervals. Also shown is the pathway of the glomerular filtrate (arrows). The filtrate passes through the endothelial fenestrae, permeates the GBM, and passes through the filtration slits to reach the urinary spaces. LD, lamina densa. (B) In the nephrotic Lamb2-/- mice, the disorganization of the GBM occurs as the distribution of the anionic sites is irregular and extends beyond the lamina densa. As a result, increased amounts of ferritin and albumin pass through the GBM. Importantly, albuminuria was detectable shortly after birth and preceded podocyte foot process effacement, the latter of which was observed approximately 2 weeks after birth.

highly charged anionic sites (10) composed of heparan sulfate proteoglycans (11) were detected in the outer and inner lighter layers of the GBM (lamina rara) with cationic reagents such as lysozyme, cationized ferritin, Alcian blue, and polyethylenimine. The latter was used in the current study by Jarad et al. (2) to assess disorganization of the GBM in laminin $\beta 2$-deficient (Lamb2-deficient) mice. For a brief period, the GBM and filtration slits enjoyed equal attention, but with the discovery of nephrin and the demonstration that an $\mathrm{mAb}$ against nephrin can cause proteinuria when administered i.v. (12) and especially with the discovery of mutations in the nephrin gene in patients with CNF (1), the pendulum swung again to the epithelial slits, and the GBM was relegated to second-class status. This is in spite of the fact that evidence from human pathology, e.g., Alport syndrome and thin basement membrane disease, has demonstrated that in at least some proteinuric states, morphologic changes are seen in the GBM in the absence of alterations in the filtration slits.

The work of Jarad et al. (2) provides important evidence that may help swing the pendulum once again to a more balanced point of view. These authors investigated the effects of ablation in mice of the major laminin subtype, $\beta 2$ (Lamb2), in the GBM. They found that these animals develop proteinuria shortly after birth and the GBM appears morphologically intact, but its laminin composition is changed to a more fetal type; the anionic sites in the lamina rara are disorganized; and the GBM is more permeable to ferritin. Of great interest is their finding that defects in the GBM occur before changes (e.g., foot process flattening) occur in the epithelium (Figure 1). Significantly, the authors have shown that the situation is different in nephrotic CD2-associated protein-null $\left(C d 2 a p^{-/-}\right)$mice, which have a primary podocyte defect and also develop proteinuria and foot process flattening but show no increase in ferritin permeability of the GBM or obvious changes in the distribution of the anionic sites in the GBM.

\section{Why has the GBM been largely ignored?}

Perhaps the answer is that it is more difficult to conceptualize the existence of restrictive pores within the GBM, which is an acellular, extracellular matrix layer organized into 3 layers - the lamina densa, lamina rara interna, and lamina rara externa (Figure 2, A and B). Like all basement membranes (or basal lamina), the GBM is composed of glycoproteins (laminins, type IV collagen, entactin/nidogen) and proteoglycans (agrin, perlecan). The lamina densa is a relatively homogeneous-appearing extracellular matrix layer, whereas the laminae rarae are less dense and contain the anionic sites defined by cationic probes. The GBM has been assumed to have the properties of a viscous gel in which the limiting pores cannot be directly visualized. On the other hand, the slit diaphragms that bridge the filtration slits are actually specialized cell junctions with properties of shallow adherens junctions that differentiate from typical junctional complexes during glomerular development (13). Like other adherens junctions, the slit diaphragms are made up of cadherins and associated catenins ( $\alpha, \beta$, and $\mathrm{p} 120$ catenins) in addition to more specialized proteins such as nephrin, NEPH1 and NEPH2 (members of the Ig family of adhesion molecules), and podocin (14). The slit diaphragms become modified into tight junctions under some pathologic conditions (e.g., puromycin aminonucleoside nephrosis) $(4,15)$. Adherens junctions function in cell-cell adhesion and are firmly attached to the actin cytoskeleton through linker proteins. In the case of the slit diaphragms, several linker proteins, including the catenins, $\mathrm{ZO}-1, \mathrm{CD} 2 \mathrm{AP}, \mathrm{CASK}$, and 

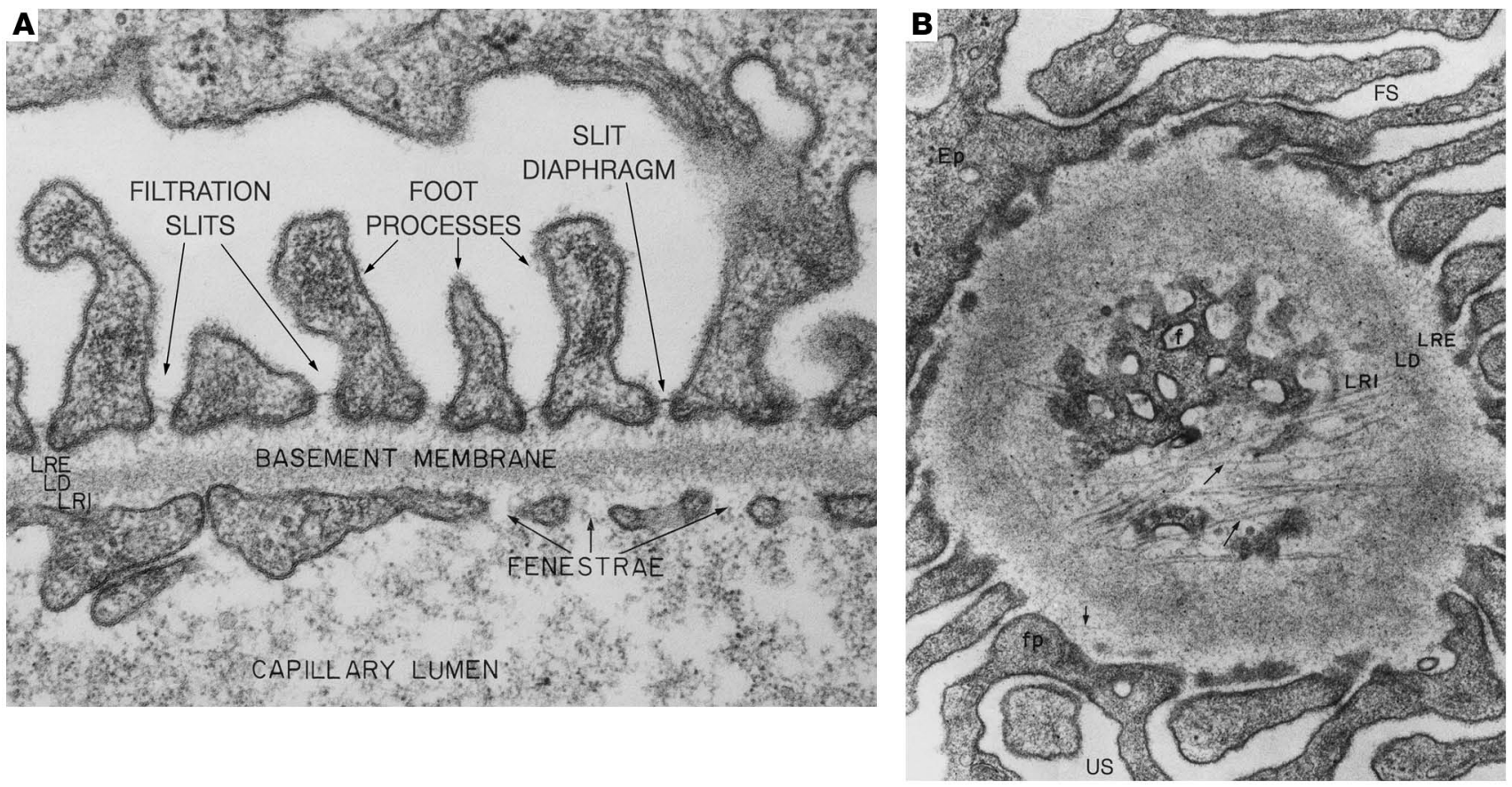

Figure 2

Electron micrographs showing a peripheral region of a glomerular capillary where filtration takes place. (A) The filtration surface consists of the endothelium, which is interrupted by fenestrae; the GBM; and the epithelial foot processes. The latter are attached to one another at their base by slit diaphragms. Note that the endothelial fenestrae are open and the GBM is directly exposed to the blood plasma. The GBM consists of 3 layers: the lamina densa and 2 lighter regions known as the lamina rara interna and lamina rara externa on either side. The lamina densa is composed of a fine $(\sim 3-\mathrm{nm})$ filamentous meshwork, which also extends across the lamina rara from the lamina densa to the endothelium and foot processes of the epithelium. Magnification, $\times 50,000$. Figure reproduced with permission from Lippincott Williams \& Wilkins (20). (B) Glomerular capillary wall cut in grazing section. In this EM view, the endothelial fenestrae (F) appear as open portholes, and the 3 layers of the GBM are cut broadly. The fine fibrils of the lamina densa meshwork extend across the lamina rara externa to the base of the podocytes' foot processes (fp, short arrow), and larger (10-nm) fibrils (long arrows) are located between the endothelium and GBM. The epithelial (Ep) filtration slits (FS) are also cut in grazing section, and the slit membranes are not detectable in this plane of section. Magnification, $\times 40,000$. US, urinary space. Figure reproduced with permission from Plenum Press (21).

MAGI-2, have been shown to be involved in this attachment (16). In the glomerulus, the slit diaphragms function to firmly attach the foot processes of the podocyte to one another on their lateral surfaces, and integrins and dystroglycans function to attach the soles of the podocyte's feet to the GBM and to maintain the organization of the anionic sites (17). Adherens junctions are not known to form permeability barriers along the intercellular spaces. This function is normally assigned to tight junctions.

\section{The alternative model of glomerular permeability: the GBM as the size-selective filter}

In spite of the fascination with the filtration slits, the model that more closely fits the various experimental data, the findings from human pathology, and now the observations in Lamb2 $2^{-/-}$mice (2), one in which the GBM represents the site of the selective barrier to proteins and the filtration slits function as a porous support that limits hydraulic flux across the glomerulus $(5,15$, 18). As pointed out long ago, if there were 2 barriers in series, one would expect to see large molecules accumulating against the GBM and small molecules in the slits, which has never been observed. Moreover, the slits would be susceptible to clogging. From calculations based on gel behavior, Smithies (19) has recently put forth a gel permeation/diffusion hypothesis that supports the concept that size-selectivity of the glomerulus resides solely in the GBM and the epithelial slits impose substantial resistance to liquid flow across the glomerulus without acting as a molecular sieve. He further proposes that the size-selective properties of the GBM are determined by permeation and diffusion.

Of particular interest in the present work (2) is the demonstration that proteinuria and changes in the anionic sites in the GBM precede foot process flattening (Figure 2).
These findings imply that the composition and organization of the GBM affect the organization of the foot processes and epithelial slits. They also fit together with the findings of Kojima et al. (17), which underline that organization of the GBM and epithelial structure are closely intertwined. These authors demonstrated that treatment with ROS and protamine, which rapidly induces foot process flattening, leads to disorder in the fibrillar components and anionic sites in the lamina rara externa of the GBM, presumably due to disruption in the linkage between $\alpha$-dystroglycan in the podocyte cell membrane and the globular domains of laminin and agrin in the GBM. Foot process flattening and the accompanying changes in the organization of the filtration slits are readily visualized by routine EM, whereas changes in the organization of the GBM are not evident and require more refined analysis of the GBM, such as that used in the current study. 
The demonstration by Jarad et al. (2) that albuminuria coincides with defects in the GBM is timely and provides key evidence pointing to GBM as the selective, molecular sieve. This work also calls attention to the limitations of routine EM in analysis of defects in the GBM and to the necessity for detailed evaluation of the permeability and organization of the GBM. Hopefully, the necessity for closer scrutiny will also eventually lead to the development of new, more sophisticated methods for analysis of the GBM.

Address correspondence to: Marilyn G. Farquhar, Department of Cellular and Molecular Medicine, University of California, San Diego, George Palade Laboratories, Room 210, 9500 Gilman Drive, La Jolla, California 92093, USA. Phone: (858) 534-7711; Fax: (858) 534-8549; E-mail: mfarquhar@ucsd.edu.

1. Tryggvason, K. 1999. Unraveling the mechanisms of glomerular ultrafiltration: nephrin, a key component of the slit diaphragm. J. Am. Soc. Nephrol. 10:2440-2445.

2. Jarad, G., Cunningham, J., Shaw, A.S., and Miner, J.H. 2006. Proteinuria precedes podocyte abnormalities in Lamb2 $2^{-/-}$mice, implicating the glomerular basement membrane as an albumin barrier. J. Clin. Invest. 116:2272-2279. doi:10.1172/JCI28414.

3. Farquhar, M.G., Wissig, S.L., and Palade, G.E. 1961.
Glomerular permeability. I. Ferritin transfer across the normal glomerular capillary wall. J. Exp. Med. 113:47-66.

4. Farquhar, M.G., and Palade, G.E. 1961. Glomerular permeability. II. Ferritin transfer across the glomerular capillary wall in nephrotic rats. J. Exp. Med. 114:699-716.

5. Caulfield, J.P., and Farquhar, M.G. 1974. The permeability of glomerular capillaries to graded dextrans. Identification of the basement membrane as the primary filtration barrier. J. Cell Biol. 63:883-903.

6. Farquhar, M.G. 1975 . The primary glomerular filtration barrier - basement membrane or epithelial slits? Kidney Int. 8:197-211.

7. Rodewald, R., and Karnovsky, M.J. 1974. Porous substructure of the glomerular slit diaphragm in the rat and mouse. J. Cell Biol. 60:423-433.

8. Ryan, G.B., and Karnovsky, M.J. 1976. Distribution of endogenous albumin in the rat glomerulus: role of hemodynamic factors in glomerular barrier function. Kidney Int. 9:36-45.

9. Brenner, B.M., Hostetter, T.H., and Humes, H.D. 1978. Glomerular permselectivity: barrier function based on discrimination of molecular size and charge. Am. J. Physiol. 234:F455-F460.

10. Caulfield, J.P., and Farquhar, M.G. 1976. Distribution of anionic sites in glomerular basement membranes: their possible role in filtration and attachment. Proc. Natl. Acad. Sci. U. S. A. 73:1646-1650.

11. Kanwar, Y.S., and Farquhar, M.G. 1979. Isolation of glycosaminoglycans (heparan sulfate) from the glomerular basement membrane. Proc. Natl. Acad. Sci. U. S. A. 76:4493-4497.

12. Orikasa, M., Matsui, K., Oite, T., and Shimizu, F. 1988. Massive proteinuria induced in rats by a single intravenous injection of a monoclonal antibody. J. Immunol. 141:807-814.

13. Reeves, W., Caulfield, J.P., and Farquhar, M.G. 1978. Differentiation of epithelial foot processes and filtration slits: sequential appearance of occluding junctions, epithelial polyanion, and slit membranes in developing glomeruli. Lab. Invest. 39:90-100.

14. Pavenstadt, H., Kriz, W., and Kretzler, M. 2003. Cell biology of the glomerular podocyte. Physiol. Rev. 83:253-307.

15. Caulfield, J.P., Reid, J., and Farquhar, M.G. 1976. Alterations of the glomerular epithelium in aminonucleoside nephrosis. Evidence for formation of occluding junctions and epithelial cell detachment. Lab. Invest. 34:43-59.

16. Lehtonen, S., et al. 2005. Cell junction-associated proteins IQGAP1, MAGI-2, CASK, spectrins, and alpha-actinin are components of the nephrin multiprotein complex. Proc. Natl. Acad. Sci. U. S. A. 102:9814-9819.

17. Kojima, K., et al. 2004. Podocyte flattening and disorder of glomerular basement membrane are associated with splitting of dystroglycan-matrix interaction. J. Am. Soc. Nephrol. 15:2079-2089.

18. Farquhar, M.G. 1978. Structure and function in glomerular capillaries. Role of the basement membrane in glomerular filtration. In Biology and chemistry of basement membranes. N.A. Kefalides, editor. Academic Press. New York, New York, USA. 43-80.

19. Smithies, O. 2003. Why the kidney glomerulus does not clog: a gel permeation/diffusion hypothesis of renal function. Proc. Natl. Acad. Sci. U. S. A. 100:4108-4113.

20. Farquhar, M.G., and Kanwar, Y.S. 1980. Characterization of anionic sites in the glomerular basement membranes of normal and nephrotic rats. In Renal pathophysiology. A. Leaf, G. Giebisch, L. Bolis, and S. Gorini, editors. Raven Press. New York, New York, USA. 57-74.

21. Farquhar, M.G., and Kanwar, Y. 1983. Functional organization of the glomerulus: presence of glycosaminoglycans (proteoglycans) in the glomerular basement membrane. In Immune mechanisms in renal disease. M. Cummings, A. Michael, and C.B. Wilson, editors. Plenum Press. New York, New York, USA. 157-174. 